\title{
ESTUDIO CUALITATIVO DE LOS CONOCIMIENTOS DE LOS ESTUDIANTES ESPAÑOLES DE EDUCACIÓN SECUNDARIA SOBRE POLÍMEROS
}

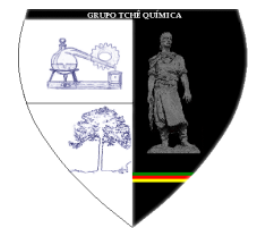

\section{A QUALITATIVE STUDY OF HIGH SCHOOL SPANISH STUDENTS' KNOWLEDGE ABOUT POLYMERS}

\author{
SERRANO-AROCA, Ángel ${ }^{1 *}$; SOLAZ-PORTOLÉS, Joan Josep ${ }^{2}$ \\ ${ }^{1}$ Universidad Católica de Valencia "San Vicente Mártir" (UCV), Facultad de Veterinaria y Ciencias \\ Experimentales, Departamento de Ciencias Aplicadas y Tecnológicas, C/ Guillem de Castro 94, 46003 Valencia \\ (Spain) \\ ${ }^{2}$ Universitat de València, Facultat de Magisteri, Departament de Didàctica de les Ciències Experimentals i \\ Socials, Avinguda dels Tarongers 4, 46022 Valencia (Spain) \\ ${ }^{*}$ Autor correspondente \\ e-mail: angel.serrano@ucv.es
}

Received 22 June 2014; accepted 29 June 2014

\section{RESUMEN}

Dada la importancia de los materiales poliméricos en la actualidad y los escasos estudios de las ideas de los estudiantes de secundaria sobre ellos, se lleva a cabo un estudio que pretende aproximarse al conocimiento de los estudiantes españoles de educación secundaria sobre estos materiales. Se ha empleado una metodología cualitativa, basada en entrevistas semi-estructuradas. Han participado 8 estudiantes de educación secundaria obligatoria ( $10^{\circ}$ grado, 16 años) y 4 estudiantes de bachillerato ( $12^{\circ}$ grado, 18 años). Los resultados revelan que: a) el nivel de conocimientos sobre polímeros de los estudiantes de educación secundaria obligatoria es bajo, esto es, no es el que corresponde a un ciudadano alfabetizado científicamente; y b) estos conocimientos no mejoran significativamente tras la formación del bachillerato científico.

Palabras clave: Enseñanza de las ciencias, ideas de los alumnos, educación media, sustancias macromoleculares, entrevistas clínicas

\begin{abstract}
Polymeric materials are important at present and there are few studies of High School students' ideas about them. Therefore, we carried out a study that aims to approach High School Spanish students' knowledge about these materials. A qualitative methodology, based on semi-structured interviews, has been used. Twelve High School students (eight of $10^{\text {th }}$ grade and four of $12^{\text {th }}$ grade) have participated in this research. Results indicate that: a) Tenth grade students' knowledge level about polymers is low, i.e. this level is inadequate for a scientifically literate citizen; and b) This knowledge level does not significantly improve after finishing $12^{\text {th }}$ grade.
\end{abstract}

Keywords: Science education, pupils' ideas, secondary education, macromolecular substances, clinical interview. 


\section{INTRODUCCIÓN}

Los polímeros son un tipo de materiales extensamente utilizados hoy en día en nuestra sociedad debido a sus únicas propiedades y a su económico coste de producción. Estos materiales son esenciales para su uso como plásticos, fibras o elastómeros con multitud de aplicaciones en transporte, embalaje, envases, construcción, comunicación, vestidos, medicina, etc. Desde el fin de la Segunda Guerra Mundial, el campo de los materiales se ha visto revolucionado por los polímeros sintéticos que han ido sustituyendo a materiales tradicionales como la madera y los metales a lo largo de las últimas décadas. Los plásticos son actualmente en nuestra sociedad, sin lugar a dudas, uno de los materiales más utilizados en todo tipo de sector industrial, destacando por su baja densidad, consistentes propiedades mecánicas, resistencia a la corrosión, gran aislante, posibilidad de fabricar piezas a medida en serie, etc.

Las publicaciones de Seymour (1988) y Gertz (1995) ponen en evidencia que los polímeros están muy presentes en todos los lugares. Por otra parte, respecto al medio ambiente, es necesario que las personas tengan un cierto nivel de conocimientos sobre polímeros para que nuestra sociedad pueda tomar decisiones responsables. De este modo, se podría fomentar un consumo responsable y lograr así reducir la cantidad de residuos. También se conseguiría fomentar la reutilización de objetos para ahorrar materia prima y ahorrar energía de producción, haciendo así un uso más racional de la energía. Por lo tanto, es evidente que todo ciudadano culto de nuestra sociedad tiene que tener unos conocimientos mínimos sobre Química en general (Pereira et al., 2013), y sobre materiales poliméricos en particular, al terminar la Educación Obligatoria.

En lo que se refiere a la didáctica, un aspecto que ha suscitado, y suscita, mucho interés entre los investigadores es el de las concepciones de los estudiantes (Sanmartín et al., 2014; Trinidad-Velasco y Garritz, 2003). Sin embargo, no se han investigado en profundidad las concepciones de los estudiantes de secundaria sobre polímeros, y solamente aparece em la bibliografía algún resultado colateral relacionado com ellos (Scheffel et al,
2009; Shwartz et al., 2006). Sin embargo, muchos proyectos de orientación cienciatecnología-sociedad (CTS) han tomado el estudio de los materiales como hilo conductor de los contenidos de Química. Por ejemplo, en el Reino Unido, el proyecto Salters Chemistry se estructuró en unidades tales como Minerales, Combustibles, Polímeros, etc. (Bennett y Lubben, 2006). Esta filosofía fue posteriormente incorporada en otros proyectos como el Science for XXI Century (Burden, 2005).

En España, el estudio de los materiales se introduce por primera vez en el currículo del área de las ciencias de la naturaleza de la ESO (Educación Secundaria Obligatoria, 12-16 años) en 1992. La experiencia de todos estos años es que ha sido difícil consolidar los materiales como un contenido básico en el currículo de ciencias de naturaleza en la ESO debido a las dificultades del tiempo disponible (Caamaño, 2009). No obstante, el estudio de los materiales en el área de ciencias de la naturaleza tiene una escasa presencia en el currículo actual (De Pro, 2007). En concreto, en el currículo de Química de Bachillerato se introducen algunos contenidos CTS relacionados con los materiales y sustancias químicas, tales como los polímeros (Camaño, 2006).

Actualmente se imparten en España contenidos sobre polímeros en dos asignaturas de la ESO (Tecnología, obligatoria a los 15 años; y Física y Química, optativa a los 16 años) y en tres de Bachillerato (Ciencias para el Mundo contemporáneo, obligatoria a los 17 años; Tecnología Industrial, optativa a los 17 años; y Química, optativa a los 18 años). Precisamente, nuestro objetivo es indagar sobre las ideas de los estudiantes sobre polímeros al acabar la ESO $\left(4^{\circ}\right.$ de la ESO, 16 años), tanto en los estudiantes que escogen el área científica como el área de humanidades y sociales; y al acabar el Bachillerato científico $\left(2^{\circ}\right.$ de Bachillerato, 18 años). Esto es, nuestro problema a investigar es: ¿tienen los estudiantes de secundaria un nivel adecuado de conocimientos sobre polimeros?

\section{METODOLOGÍA}

El diseño de la investigación es cualitativo y se utiliza la entrevista clínica como técnica de recogida de información. La entrevista clínica se 
ha revelado como un instrumento de gran valor en la investigación educativa, particularmente en la investigación en la didáctica de las ciencias, y en especial en el área de las concepciones de los estudiantes (Moreira y Lang da Silveira, 1993). Nosotros haremos uso de entrevistas semi-estructuradas (Mayan, 2001).

\subsection{Sujetos participantes}

Han participado 12 alumnos de un centro de educación secundaria público de la ciudad de València (España). De ellos, 8 son del último curso de la ESO y tienen 16 años (la mitad son de ciencias, cursaron la asignatura Física y Química en ese año y pertenecían al mismo grupo; la otra mitad son de humanidades y sociales y pertenecían a un grupo distinto). Los 4 restantes son del último curso de Bachillerato y tienen 18 años (todos ellos cursaron la asignatura de Química y pertenecen al mismo grupo). Los 4 estudiantes de cada uno de los 3 grupos fueron seleccionados atendiendo a sus resultados académicos a lo largo del curso: 1 con buenos resultados, 1 con malos resultados y 2 con resultados intermedios.

\subsection{Procedimiento}

La realización de las entrevistas a los alumnos se ha llevado a cabo siguiendo un guión de preguntas (que no se hacen necesariamente en el orden que están escritas) expuestas en la Tabla 1. Como puede verse en esta tabla las preguntas están relacionadas con 10 cuestiones básicas sobre polímeros: concepto de polímero, concepto de polimerización, propiedades de los plásticos, objetos que contienen polímeros, polímeros naturales, plásticos, ecología y plásticos, PET, plásticos biodegradables y polímeros conductores. Las 10 cuestiones básicas sobre polímeros fueron seleccionadas por 2 profesores universitarios y 1 profesor de educación secundaria, de acuerdo con su importancia en la educación secundaria, a partir de una batería de 25 cuestiones.

Tabla 1. Preguntas que se formularon durante las entrevistas en cada una de las 10 cuestiones sobre polímeros.

P-1.-Concepto de polímero: ¿Qué es un monómero y qué es un polímero? ¿Qué tipos de polímeros conoces? Dime algunos ejemplos de nombres de polímeros que conozcas. ¿Te han enseñado alguna vez en clase diferentes tipos de polímeros?

P-2.-Concepto de polimerización: ¿Qué es una reacción de polimerización? ¿Has realizado o visto alguna reacción de polimerización. ¿Serías capaz de formular la reacción de polimerización del eteno (o etileno)? ¿Cómo se llama el polímero que se obtiene?

P-3.-Propiedades de los plásticos: ¿Cuáles son las propiedades únicas que poseen los plásticos que hace que se utilicen para multitud de aplicaciones? ¿Qué importancia social y económica tienen?

P-4.-Objetos que contienen polímeros: ¿Puedes enumerar 5 objetos que contengan alguna pieza de polímero que podrías encontrar en tu casa?

P-5.-Polímeros naturales: ¿Qué polímeros naturales conoces?.

P-6.- Plásticos: ¿En qué consiste la inyección y la extrusión de plásticos? ¿Para qué se utiliza?

P-7.- Ecología y plásticos: ¿Eres consciente de lo importante que son las tres erres (3R) de ecología (reducir, reutilizar y reciclar) en el caso de los plásticos? ¿Por qué?

P-8.- PET: Algunos recipientes como las botellas de agua llevan el siguiente símbolo grabado:

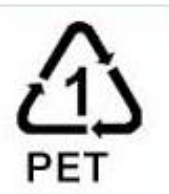

¿Sabes lo que significa el triángulo con flechas, el número y la palabra PET?

P-9.- Plásticos biodegradables: ¿Qué es un plástico biodegradable? ¿Qué ventaja presentan respecto a los plásticos normales?

P-10.- Polímeros conductores: En el año 2000 dos científicos americanos y uno japonés descubrieron los polímeros conductores. ¿Cuál es la importancia de dicho descubrimiento? ¿Sabes si este descubrimiento recibió algún premio?

Las entrevistas fueron registradas con una grabadora. Durante la entrevista se permitió 
al entrevistado la realización de matices en sus respuestas que pudieran proporcionar un valor añadido a la información que dan. $A$ continuación, fueron transcritas en un documento y analizadas por los autores de este trabajo. Se pudieron encontrar hasta cuatro categorías diferentes de respuestas en cada una de las 10 cuestiones básicas sobre polímeros: correcta, casi correcta, poco correcta, incorrecta. Finalmente un autor de este trabajo y un profesor universitario (es decir, dos jueces) evaluaron las respuestas de los estudiantes y las clasificaron dentro de alguna de las cuatro categorías anteriomente citadas. El cálculo del coeficiente kappa de Cohen para evaluar el grado de acuerdo entre jueces arrojó un valor de 0.85 , lo que indica un muy buen grado de acuerdo. Las discrepancias fueron resueltas de mutuo acuerdo.

\section{RESULTADOS Y DISCUSIÓN}

Se representan gráficamente en la Figura 1 los resultados obtenidos en las entrevistas clínicas a los alumnos de $4^{\circ}$ de la ESO de ciencias.

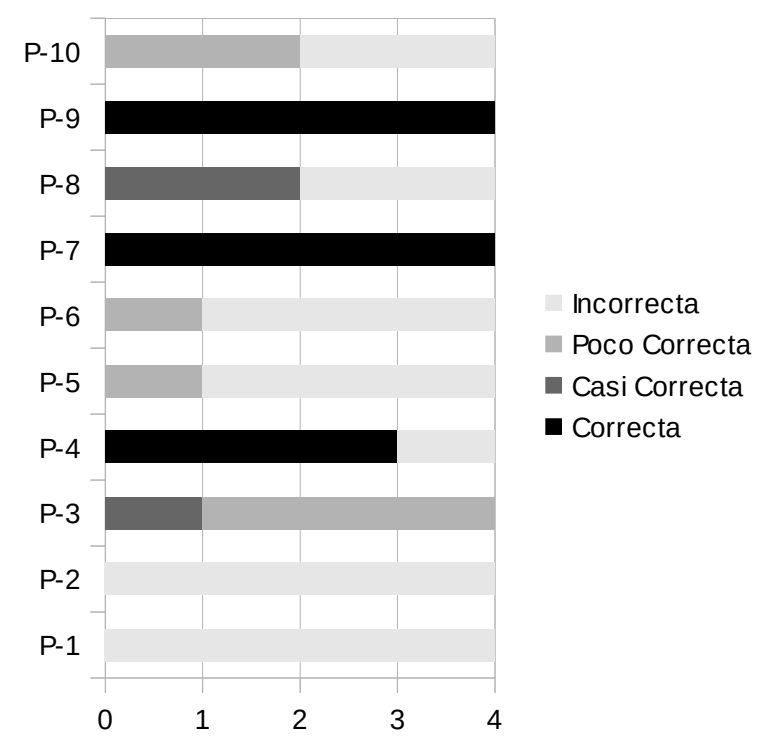

Figura 1. Número de estudiantes de $4^{\circ}$ de la ESO (16 años) de ciencias en cada categoría de respuesta en las diez preguntas de la Tabla 1 $(P-1, P-2$, etc.).
Frente a la tan básica e importante pregunta de definir lo que es un monómero y un polímero $(\mathrm{P}-1)$, la respuesta de todos los alumnos fue incorrecta. Una estudiante ofreció la mejor respuesta, aunque incorrecta, afirmando que "un polímero es aquello que llamamos comúnmente plástico". No supo definir monómero ni dar más información sobre la cuestión. Otros dos alumnos asociaron también monómero y polímero con la palabra plástico pero de forma más incorrecta. Por último, hubo otro alumno que confundió monómero y polímero con monomio y polinomio. Así pues parece que hay una tendencia en estos estudiantes, que recordemos tienen 16 años y acaban la educación secundaria obligatoria, a pensar erróneamente que polímero es sinónimo de plástico, cuando los plásticos son únicamente uno de los tipos de polímero que existen.

Nuevamente la estudiante del grupo consiguió la mejor respuesta a la P-3 aunque se quedó en la categoría de respuesta casi correcta: "los plásticos no son conductores, son de fácil fabricación, se pueden moldear fácilmente (depende del tipo). Son duraderos y algo resistentes a las temperaturas. Abaratan el precio del producto, evitan la electrocución, son duraderos y cómodos".

La mayoría de los alumnos supieron enumerar cinco objetos que contengan alguna pieza de polímero que podrían encontrar en su casa ( $P-4)$ pero sin saber distinguir claramente entre los tipos de polímeros (plásticos, elastómeros, fibras, etc.). Sin embargo, la mayoría de los estudiantes no conocen ningún polímero natural (P-5).

Respecto a la pregunta sobre la inyección y extrusión de plásicos (P-6), tres de los cuatro alumnos no supieron contestarla y un estudiante dio una respuesta muy pobre con una visión algo deformada: "la inyección de plásticos es el bombear el plástico hasta que conseguimos una pieza grande con aire dentro". En cuanto a la P8 , ningún estudiante supo dar la respuesta correcta. La mejor respuesta fue: "el triángulo con flechas indica que el producto es reciclable. EI número indica a que grupo pertenece. PET significa que este producto no deja residuos".

En la $\mathrm{P}-10$, relativa a los polímeros conductores, dos ejemplos de respuestas son: a) 
"la importancia de este descubrimiento es el poder utilizar los polímeros para conducir el calor, la electricidad, etc.(no lo tengo claro)" ; y b) "no lo sé, pero los cables de los electrodomésticos están cubiertos por plástico para que no recibamos corriente, así que lo veo un poco peligroso si se usara para eso". Ninguno de los estudiantes sabía que se concedió un Premio Nobel de Química por este descubrimiento.

La representación de los resultados de las entrevistas clínicas a los alumnos de $4^{\circ}$ de la ESO de humanidades y ciencias sociales se ofrece en la Figura 2.

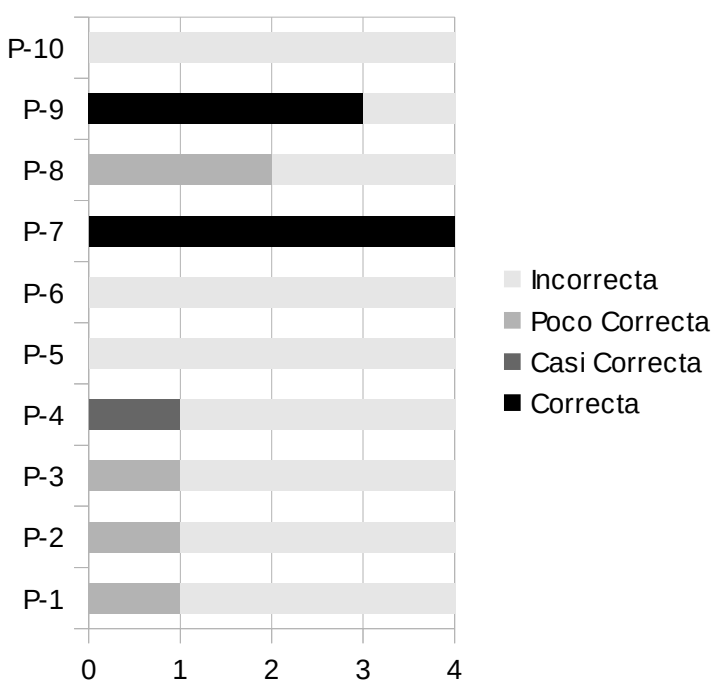

Figura 2. Número de estudiantes de $4^{\circ}$ de la ESO (16 años) de humanidades y ciencias sociales en cada categoría de respuesta en las diez preguntas de la Tabla 1(P-1, P-2, etc.).

Estos resultados son bastante parecidos a los de los estudiantes del anterior grupo de ciencias, aunque algo peores. Como puede verse, $\mathrm{P}-7$ y $\mathrm{P}-9$ son las que mejor se responden tanto en este grupo con en el anterior. Sin embargo, en las respuestas a P-3 y P-4 hay más estudiantes de ciencias que se aproximan más a las correctas que de humanidades y ciencias sociales.

La mejor respuesta a la P-1 fue: "son los materiales que forman el plástico como las bolsas. Son moléculas formadas por células que se repiten". Respecto a las propiedades únicas de los plásticos y su importancia social y económica (P-3), sólo un estudiante fue capaz de dar una respuesta de categoría poco correcta. En la P-8, únicamente un alumno supo decir de forma correcta que el triángulo con flechas significa que se puede reciclar el material, y también sólo uno que PET era el nombre del plástico.

Finalmente, se muestran los resultados obtenidos en las entrevistas clínicas a los alumnos de $2^{\circ}$ de Bachillerato en la representación gráfica siguiente (Figura 3).

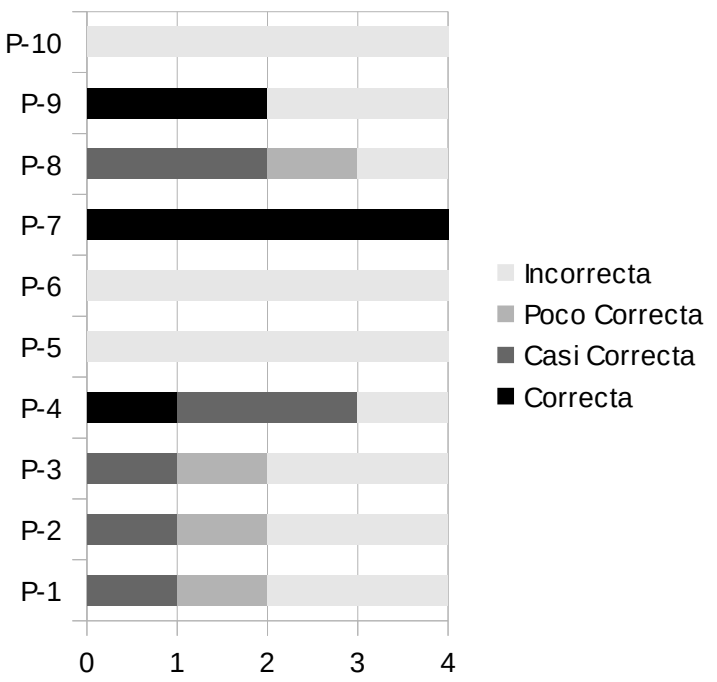

Figura 3. Número de estudiantes de $2^{\circ}$ de Bachillerato científico (18 años) en cada categoría de respuesta en las diez preguntas de la Tabla 1(P-1, P-2, etc.).

Puede apreciarse que mejoran un poco las respuestas, en relación a los estudiantes de la ESO de ciencias, en P-1, P-2 y P-8; las demás son iguales o incluso un poco peores, como el caso de P-5, P-6 y P-9.

En P-1, también los estudiantes de este nivel toman polímero como sinónimo de plástico. Una estudiante acertó a decir que: "Ios polímeros están formados por componentes denominados monómeros". La mejor respuesta fue: "un monómero es una sustancia que se agrupa con otras de las mismas características para formar polímeros. Un polímero es un conjunto de 
monómeros que se han modificado para obtener unas características determinadas. Lo he visto en el programa de televisión el Hormiguero. Son polímeros el PVC, el PET y otros plásticos, por ejemplo".

La mitad de los alumnos no sabe explicar lo que es una reacción de polimerización (P-2). La mejor respuesta obtenida: "Es una reacción mediante la cual se obtienen polímeros, conseguidos haciendo reaccionar monómeros a temperaturas y presiones determinadas para obtener ciertas propiedades. No, no he realizado polimerizaciones en el centro en el que estudio aunque sí sinteticé nylon en una visita a la Universidad. No soy capaz de formularla".

En la P-8 dos estudiantes consiguieron acercarse a la respuesta correcta. Uno de ellos dijo: "el número es el grado de dureza del plástico, las flechas es que es reciclable y lo de PET no lo sé. No sabía que estaban grabados esos símbolos". Los otros dos alumnos restantes dieron respuestas incorrectas.

\section{CONCLUSIONES}

De los resultados obtenidos en este estudio cualitativo pueden extraerse como mínimo dos conclusiones, siempre teniendo presentes las limitaciones de nuestra investigación (pocos sujetos y del mismo centro educativo) que imposibilitan efectuar generalizaciones con márgenes de fiabilidad elevados. En nuestra opinión, la conclusión más importante es que el nivel de conocimientos sobre materiales poliméricos adquirido por los estudiantes entrevistados al acabar la educación secundaria obligatoria (16 años) está muy lejos de ser el óptimo. Si uno de los objetivos en la educación secundaria obligatoria es la alfabetización científica de la ciudadanía (De Oliveira et al., 2013), creemos que los conocimientos puestos de manifiesto por nuestros estudiantes sobre polímeros evidencian que no estamos proporcionando la formación adecuada a nuestros ciudadanos para conocer mejor el mundo que les rodea, ni para ser consumidores críticos y responsables, ni para ser activistas en favor del medio ambiente.

Por otra parte, la segunda conclusión hace referencia a la enseñanza que se lleva a cabo en el Bachillerato científico (16-18 años). Hemos constatado que durante ese período de formación los estudiantes no han mejorado significativamente sus conocimientos sobre materiales poliméricos, respecto de la fase académica anterior, a pesar de tratarse de un contenido de especial relevancia para la ciencia contemporánea y estar incluido en el currículo.

Hemos de destacar que en un estudio realizado en Israel con estudiantes entre el grado 10 y el grado 12 (es decir, entre 16 y 18 años) sobre el nivel de alfabetización química (Shwartz, et al., 2006), aparece un ítem sobre el concepto de polímero en el que el $37 \%$ de estudiantes de grado 10 y el $27 \%$ en el grado 12 afirma desconocer qué es un polímero. En consecuencia, parece que la conclusiones de este trabajo están en línea com lo que ocurre en otros países.

Finalmente, señalaremos que, en nuestra opinión, las posibles causas de este bajo nivel de conocimientos sobre polímeros en la educación secundaria han de buscarse fundamentalmente en el diseño curricular y en la metodología de enseñanza. En el primer caso, pensamos que más contenidos sobre sustancias poliméricas han de impregnar de manera transversal el currículum de la educación secundaria, destacándose sus propiedades. En el segundo caso, todo apunta a que una metodología activa donde el estudiante construye sus conocimientos de manera colaborativa puede mejorar los resultados académicos (Acar \& Tarhan, 2006; Furió \& Furió, 2009)

\section{AGRADECIMENTOS}

Agradecemos a los profesores $y$ estudiantes del Instituto de Educación Secundaria Isabel de Villena de Valencia su amable participación en este estudio.

\section{REFERENCIAS}

1. Seymour, R.B. Journal of Chemical Education, 1988, 65, 327.

2. Gertz, S. Polymers All Around You. American Chemical Society, 1995, Washington, DC.

3. Pereira, C.E., Oliveira, N., Benedetti Filho, E., Fionucci, A.R. (2013). Periódico Tchê Química, 2013, 10, 12. Disponível em www.periodico.tchequimica.com, accesado en 7/06/2014. 
4. Sanmartín, B., Solaz-Portolés, J.J., Sanjosé, V. Educación Química, 2014, 25, 139.

5. Trinidad-Velasco, R., Garritz, A. Educación Química, 2003,14, 72-85.

6. Scheffel, L., Brockmeier, W., Parchmann, I. Historical material in macro-micro thinking: Conceptual change in chemical education and the history of chemistry. In J.K. Gilbert \& D. Treagust (Eds.), Multiple representations in Chemical Education, 2009, 215-250, Springer, Dordrecht.

7. Shwartz, Y., Ben-Zvi, R., Hofstein, A. Chemistry Education Research and Practice, 2006, 7, 203.

8. Bennett, J., Lubben, F. International Journal of Science Education, 2006, 28, 999.

9. Burden, J. Alambique. Didáctica de las Ciencias Experimentales, 2005, 46, 68.

10. Caamaño, A. Alambique. Didáctica de las Ciencias Experimentales, 2009, 59, 9.
11. De Pro, A. Alambique. Didáctica de las Ciencias Experimentales, 2007, 53, 3.

12. Caamaño, A. Educación Química, 2006, $17,2$.

13. Moreira, M. A., Lang de Silveira, F. Instrumento de pesquisa em ensino e aprendizagem, EDIPURCS, 1993, Porto Alegre.

14. Mayan, M.J. (2001) An introduction to qualitative methods: A training module for students and professionals, Qual Institute Press, 2001, Edmonton, Canada.

15. De Oliveira, D., Delourdes, M., Pereira, R., Vázquez-Alonso, A. Revista Electrónica de Enseñanza de las Ciencias, 2013, 12, 313.

16. Acar, B., Tarhan, L. International Journal of Science and Mathematics Education, 2006, 5, 343.

17. Furió, C., Furió, C. Educación Química, 2009, 20, 246. 